\title{
Assessment of Adverse Drug Reactions Associated with Cardiovascular Agents and Antibiotics at a Tertiary Care Hospital
}

\author{
Armin Eisa Zaei*, Siavash Shahbazi Nia and Shadan Modaresahmadi \\ Department of Pharmacy Practice, Al Ameen College of Pharmacy, Bangalore - 560027, Karnataka, India; \\ armin.eisazaei93@gmail.com
}

\begin{abstract}
The assessment of Adverse Drug Reactions (ADRs) has become increasingly significant because of the introduction of a large number of potent toxic chemicals as drugs in the last two or three decades. Therefore, it is important that continuous monitoring of adverse drug reactions in hospitals could prevent serious consequences and unexpected effects of the drugs and improve the quality of treatment in patients. Antibiotics and cardiovascular agents are the most commonly prescribed drugs which are also involved with the highest number of adverse drug reactions in the hospitals. The objective of this study was to monitor and evaluate the adverse drug reactions caused by cardiovascular agents and antibiotics at a tertiary care hospital in Bangalore, for a period of 12 months between March "2017 to March 2018". All in-patients of both genders who experienced an ADR during their hospital stay were enrolled for the study and exclusion criteria were patients admitted to hospital and did not experience an ADR. Out of 110 Adverse drug reactions, 57 (51.81\%) were caused by cardiovascular agents and 53 (48.18\%) were caused by antibiotics. Among the cardiovascular agents ADRs the most frequently reported ADRs were caused by furosemide 19 (33.33\%) followed by telmisartan $5(8.77 \%)$. Out of $53(48.18 \%)$ ADRs caused by antibiotics, $8(15.09 \%)$ were induced by ceftriaxone, followed by 7 (13.20\%) piperacillin/tazobactam, $6(11.32 \%)$ cefoperazone, $5(9.43 \%)$ levofloxacin. The most common organ system affected was the gastrointestinal system with 31 (28.18\%) followed by fluid and electrolytes 28 (25.45\%), dermatological $21(19.09 \%)$ and cardiovascular 7 (6.36\%). The causality assessment of the ADRs was carried out using the Naranjo's Scale algorithm and the majority of the ADRs were found to be possible $44(40 \%)$. Antibiotics and cardiovascular agents are the most commonly prescribed medicines in hospitals, so it is important to notice the physicians with latest adverse drug reactions of these medications. This approach consequently reduces the chances of patient harm and also improves the patient's quality of treatment, reduces the cost burden and hospital stay and minimizes the antibiotics resistance due to unresponsiveness of medications in patients.
\end{abstract}

Keywords: Adverse Drug Reactions, Antibiotics, Cardiovascular Agents, Pharmacovigilance

\section{Introduction}

According to WHO's definition an Adverse Drug Reaction (ADR) is a response to a drug that is noxious, unintended and occurs at doses normally used in human for the prophylaxis, diagnosis, and treatment of disease, or for modification of physiological function ${ }^{1,2}$.

The detection of Adverse Drug Reactions (ADRs) has become increasingly significant because of the introduction of a large number of potent toxic chemicals as drugs

${ }^{*}$ Author for correspondence 
in the last two or three decades. WHO has intervened seriously in the matter and established an international adverse drug reactions monitoring centre at Uppsala, Sweden which is collaborating with national monitoring centres in around 70 countries ${ }^{3}$.

According to a study carried out at a private tertiary care hospital in South India, the incidence of ADRs was found to be $1.8 \%$, out of which $12 \%$ of suspected ADRs were severe and $49 \%$ ADRs were moderate in severity ${ }^{4}$. Another study carried out in a tertiary care referral center in South India showed that admissions due to ADRs accounted for $0.7 \%$ of total admissions and deaths due to ADRs accounted for $1.8 \%$ of total ADRs 5 .

Therefore it is important that continuous monitoring of adverse drug reactions in hospitals could prevent such serious consequences and unexpected effects of the drugs to the patients and improve the quality of treatment in patients.

The objective of this study was to monitor and evaluate the adverse drug reactions caused by cardiovascular agents and antibiotics at a tertiary care hospital in India.

\section{Materials and Methods}

\subsection{Study Design}

This was a prospective, cross-sectional study conducted at a tertiary care hospital in Bangalore, India for a period of 12 months between March "2017 to March 2018”.

\subsection{Inclusion/Exclusion Criteria}

All in-patients, from both genders and all age groups, with a hospitalization period of at least 72 hours who experienced an ADR attributed to the cardiovascular agents and antibiotics were included in the study. All outpatients of the hospital and those who had been admitted to the Obstetrics and Gynecology Department (OBG) were excluded from this study.

\subsection{Sampling and Data Collection}

During the study period, on a daily basis, the research investigators attended the different wards of the hospital to collect the required data from the patient case sheets. The data collected include the patient's initials, age and sex; brief description of the suspected adverse drug reaction, and the onset/stop date of its occurrence; name of the suspected medications, and its indication, start date and stop date, dose, dosage form and frequency; past and present medical history of the patients, concomitant medications, and relevant tests and laboratory data. The ADRs were detected and monitored by interviewing the patients, reviewing the laboratory tests and medical charts and consulting the physicians about the patients' clinical problems. For each detected ADR a yellow form, the form used for ADR reporting to National Pharmacovigilance Centre, was filled and it is documented.

\subsection{Ethical Considerations}

The study was ethically approved by the institutional review board of the hospital. For using each patient's information in this study, each patient, care giver or parent was clearly explained about the study and signed informed consent form was obtained from him or her.

\subsection{Analysis of ADRs}

All the collected data were tabulated and analyzed to identify the probability of the reactions. To appraise this parameter, Naranjo scale algorithm has been used.

\section{Results}

Out of 2,542 patients admitted during the study period, overall 110 ADRs were reported. Out of 110 Adverse drug reactions, 57 (51.81\%) were caused by cardiovascular agents and $53(48.18 \%)$ were caused by antibiotics. ADRs were mostly occurred in females $65(59.09 \%)$ as compared to males 45 (40.90\%). Majority of patients who experienced ADRs belonged to the age group of 21-50 years. The distribution of patients with respect to their age is presented in Table 1.

Table 1. Distribution of patients with respect to age $(\mathrm{N}=110)$

\begin{tabular}{|l|l|l|}
\hline SL. NO. & AGE RANGE (YEARS) & RESULT \\
\hline 1 & $1-10$ & $2(1.8 \%)$ \\
\hline 2 & $11-20$ & $8(7.2 \%)$ \\
\hline 3 & $21-30$ & $18(16.3 \%)$ \\
\hline 4 & $31-40$ & $31(28.1 \%)$ \\
\hline 5 & $41-50$ & $25(22.7 \%)$ \\
\hline 6 & $51-60$ & $12(10.9 \%)$ \\
\hline 7 & $61-70$ & $8(7.2 \%)$ \\
\hline 8 & $71-80$ & $5(4.5 \%)$ \\
\hline 9 & $81-90$ & $1(0.9 \%)$ \\
\hline
\end{tabular}


Among the 57 (51.81\%) ADRs caused by cardiovascular agents, the most frequent reported ADRs were caused by furosemide 19 (33.33\%) followed by telmisartan 5 (8.77\%) and ramipril 5 (8.77\%) and amlodipine 4 (7.01\%). Details are shown in Figure 1.

Out of 53 (48.18\%) ADRs caused by antibiotics, 8 (15.09\%) were induced by ceftriaxone, followed by 7 (13.20\%) piperacillin/tazobactum, 6 (11.32\%) cefoperazone, 5 (9.43\%) levofloxacin, 5 (9.43\%) clindamycin and 4 (7.54\%) amoxicillin. Details are shown in Figure 2.

Among 57 ADRs induced by cardiovascular agents, 10 (17.5\%) ADRs were caused by orally administered agents and the remaining 47 (82.5\%) ADRs were caused by intravenously administered agents and among 53 ADRs caused by antibiotics, $6(11.3 \%)$ were induced by orally administered agents while the remaining 47 (88.7\%) ADRs were caused by intravenously administered antibiotics.

The most common organ system affected was the gastrointestinal system with $31(28.18 \%)$ ADRs with symptoms of diarrhea 10 (11\%), vomiting 4 (3.63\%) and abdominal pain $3(2.72 \%)$. The other organ systems affected were 28 (25.45\%) fluid and electrolytes, 21 (19.09\%) dermatological, and 7 (6.36\%) cardiovascular. Details are shown in Figure 3.

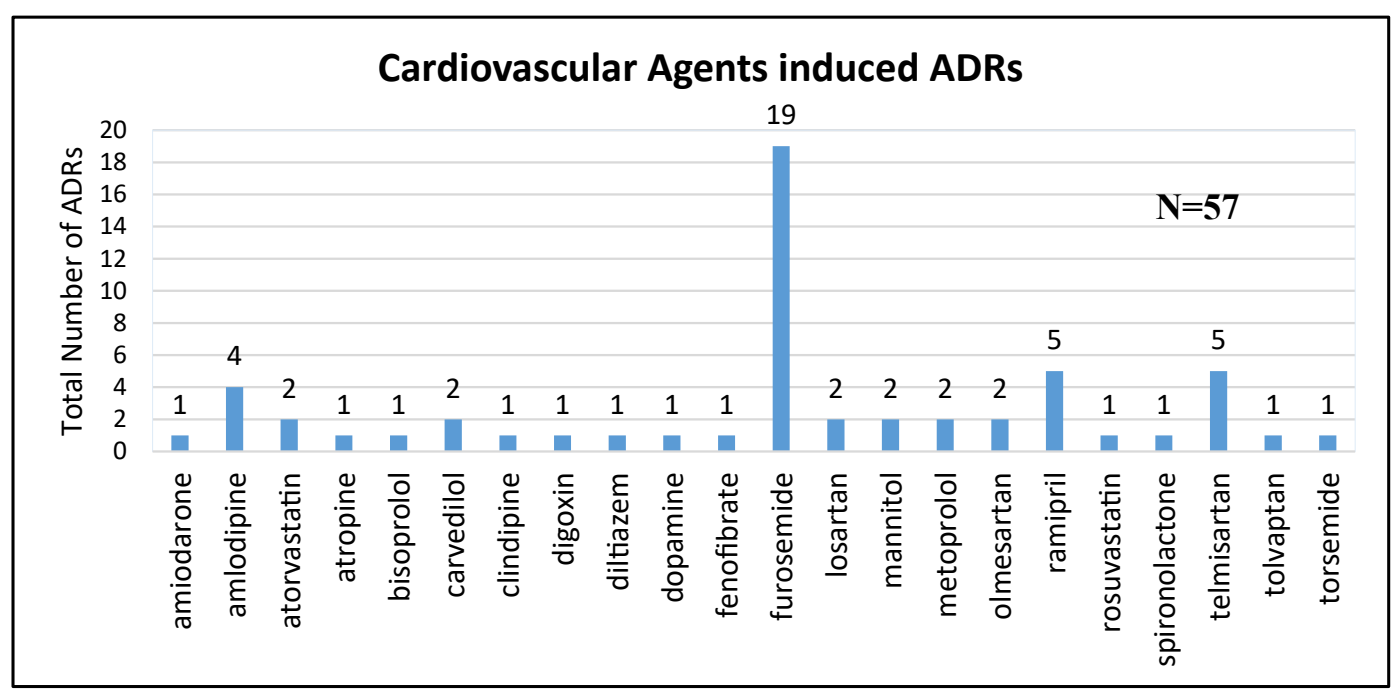

Figure 1. Cardiovascular agents involved with ADRs.

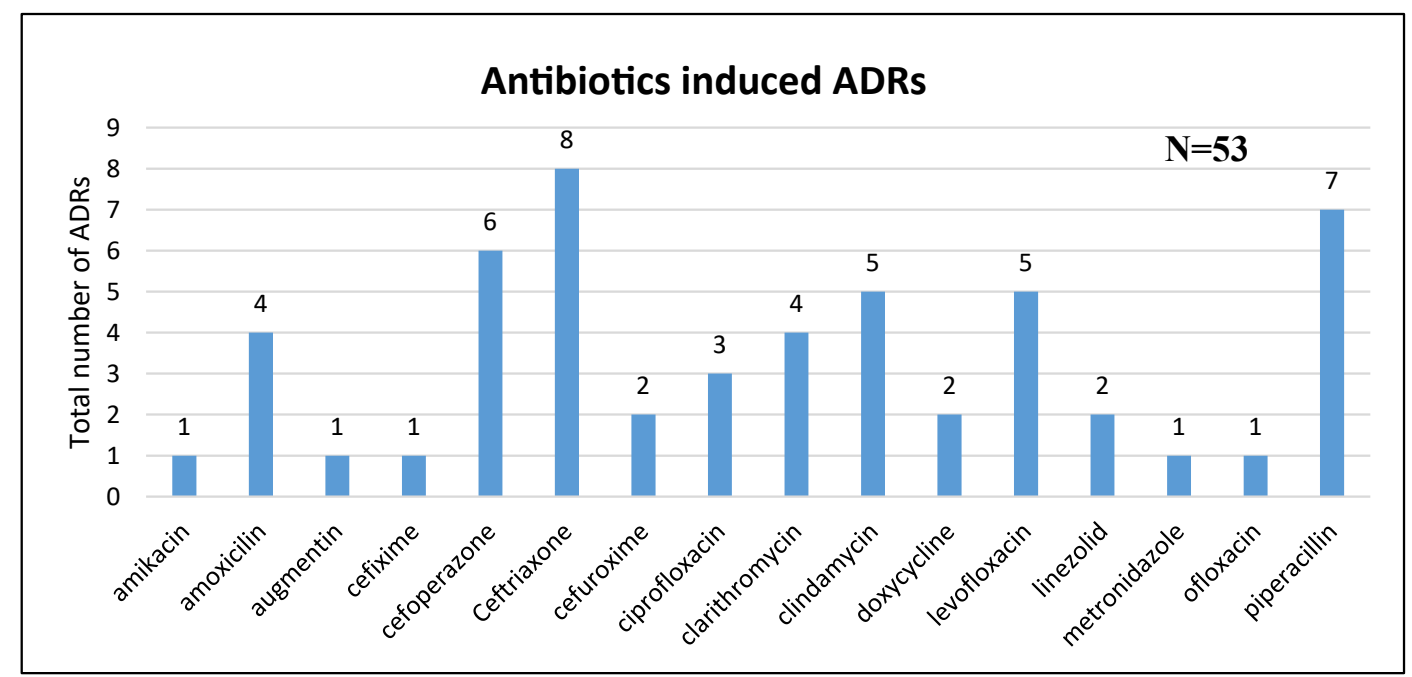

Figure 2. Antibiotics involved with ADRs 


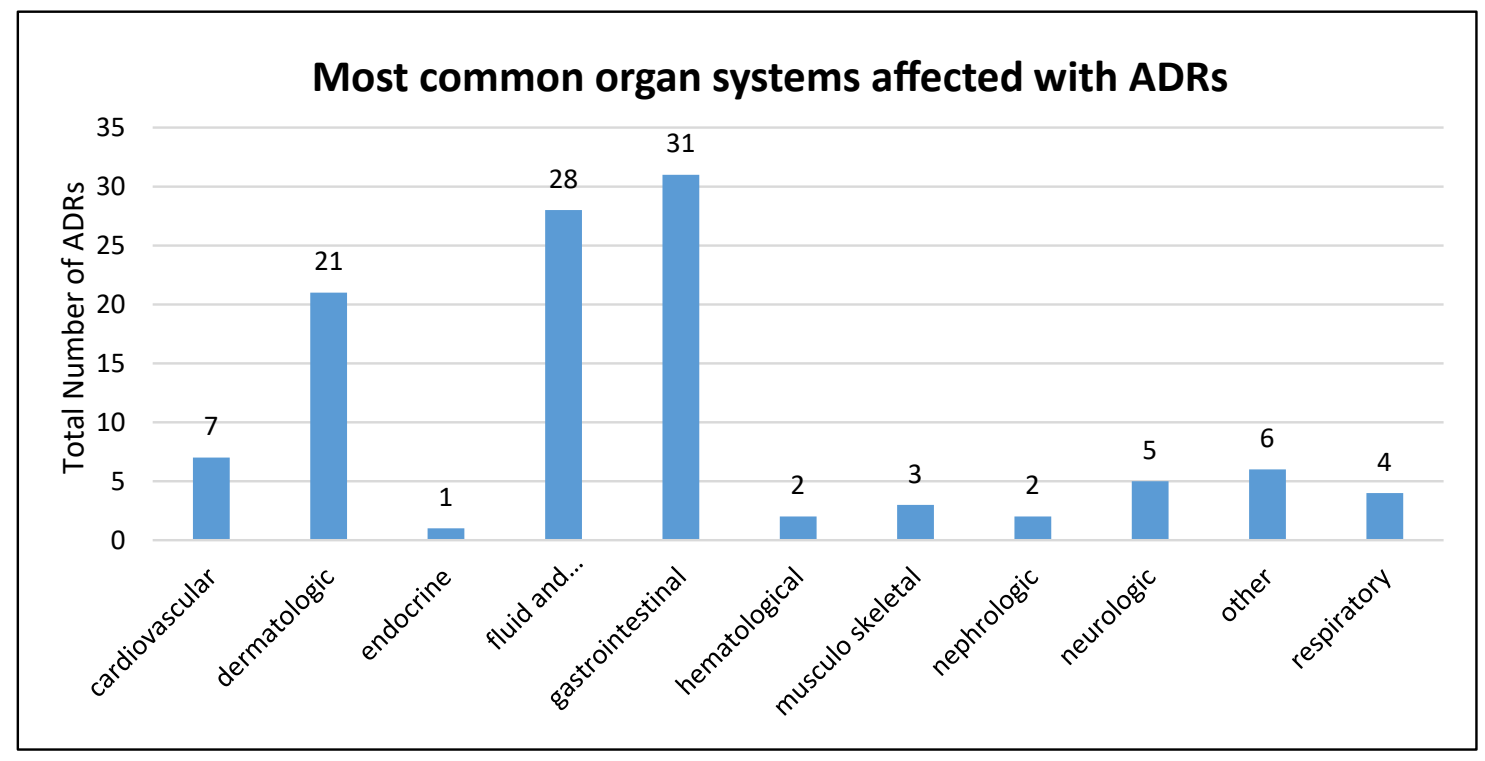

Figure 3. Most common organ systems affected with ADRs

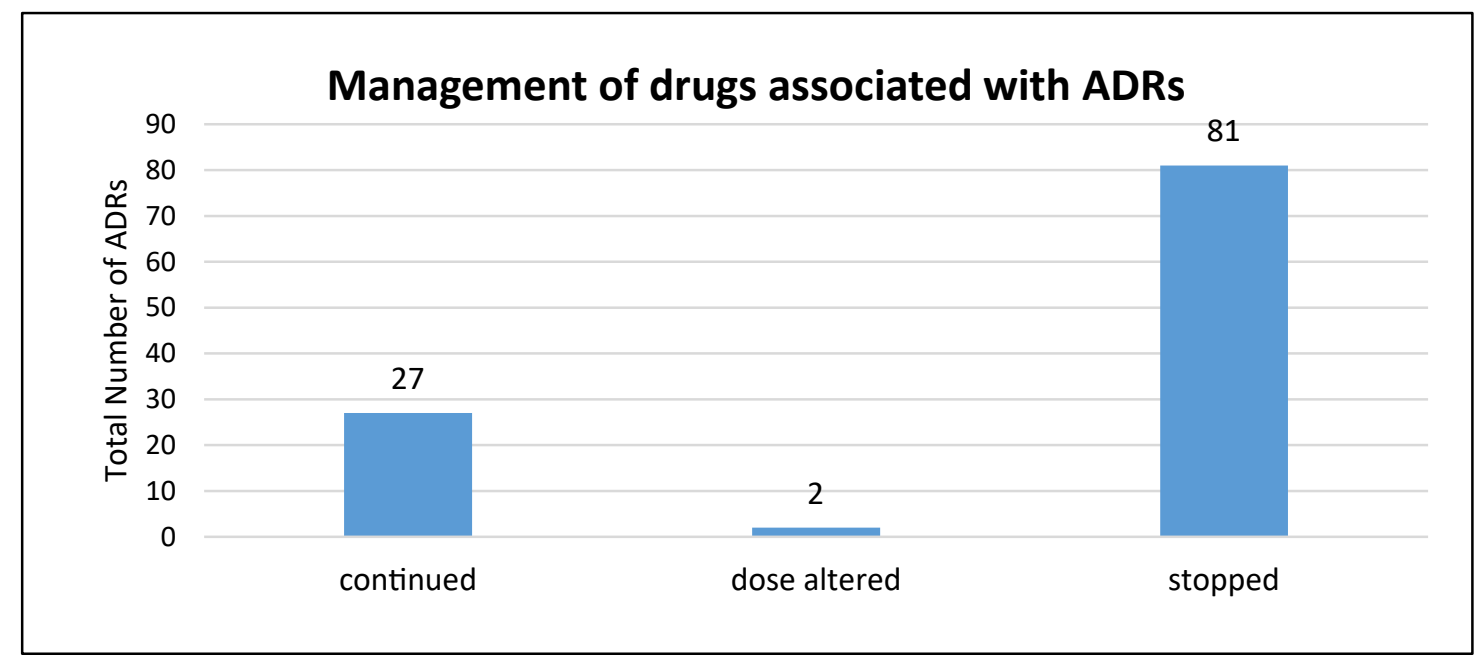

Figure 4. Management of drugs associated with ADRs.

Majority of the ADRs [81 (73.63\%)] were managed by withdrawing the suspected drugs, followed by 27 $(24.54 \%)$ continuing the same medicine and 2 (1.81\%) were managed by altering the dose of medication. Details are shown in Figure 4.

The causality assessment of the ADRs was carried out using the Naranjo's scale algorithm and the majority of the ADRs were found to be possible 44 (40\%). Details are shown in Figure 5.

\section{Discussion}

Cardiovascular agents and antibiotics were among the most commonly prescribed medicines in the hospital. As a result, the ADRs caused by these agents were also among the most commonly occurred ADRs in the hospital. It indicates that cardiovascular agents and antibiotics are the most potent drugs to cause adverse drug reactions in the hospital and if the occurred potential ADRs are 


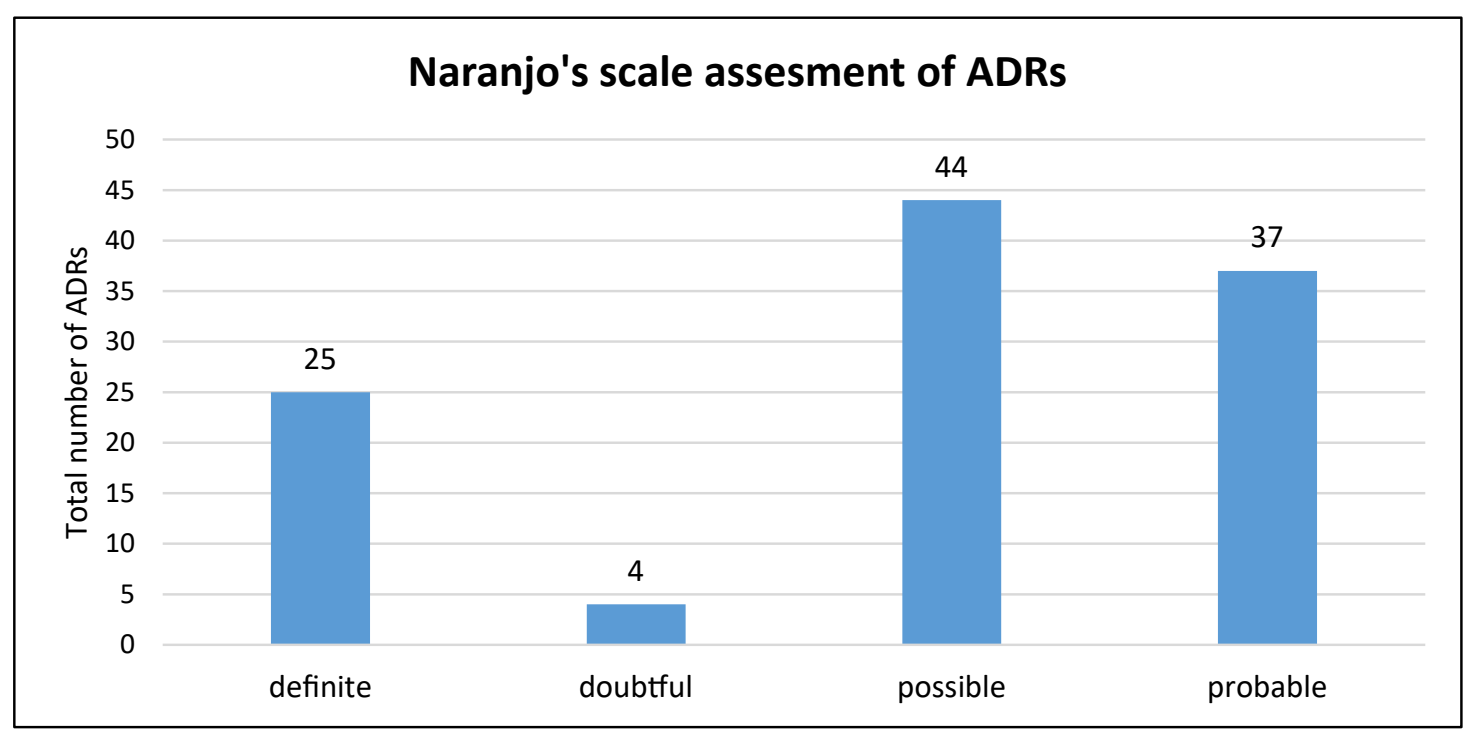

Figure 5. Causality assessment of ADRs using Naranjo's scale.

not prevented or managed, they may lead to temporary or permanent harm to the patients. The study conducted by paudel et al. and Eisazaei et al., ${ }^{6,7}$ showed the similar results to our study. Further, it proves that it is an important alert for all healthcare team members responsible for prescribing to administration and monitoring of medications for rationally prescribing of such medications. In our study, most of the adverse drug reactions affected the gastrointestinal system. This was different from the results of the study conducted by Khurshid et al. ${ }^{8}$ in which central nervous system was the most commonly affected organ system.

The causality assessment of the ADRs was carried out using the Naranjo's scale algorithm and the majority of the ADRs were found to be possible. The results of the study were similar to the results of the study conducted by Paudel $e t$ al. ${ }^{6}$

Majority of the ADRs were managed by withdrawing the suspected drugs, followed by continuing the same medicine and altering the dose of medication. One of the limitations of this study was that there was not enough data for collaboration of clinical pharmacists with physicians for management of ADRs. This could be an opportunity for more research in the region, specifically in India to evaluate the impact of clinical pharmacists collaboration with physicians in assessment and management of ADRs. This will consequently improve the patients quality of life and enhance the involvement of clinical pharmacists in hospitals.

\section{Conclusion}

As the present study relates to ADR profile of cardiovascular agents and antibiotics, it is important to notice the physicians with latest adverse drug reactions of most commonly prescribed medicines in hospitals. This approach consequently reduces the chances of patient harm and also improves the patient's quality of treatment, reduces the cost burden and hospital stay. Prevention and continues monitoring of adverse drug reactions in hospitals can be achieved by the presence of well-trained clinical pharmacists in all hospitals.

\section{References}

1. WHO. International drug monitoring the role of national centres. WHO Technical Report Series No. 498; 1972. p. 498.

2. Couper MR, Mehta DK, editors. WHO Model Formulary 2002. 1st ed. Geneva: World Health Organisation; 2002. p. 9.

3. Kurokawa T, Correa-Nunes AM, Czarnecki A. Guidelines for setting up and running a pharmacovigilance centre. Sweden: Uppsala Monitoring Centre, WHO Collaborating Centre for International Drug Monitoring; 2000. p. 4-10.

4. Sriram S, Ghasemi A, Ramasamy R, Devi M, Balasubramanian R, Ravi TK, et al. Prevalence of adverse drug reactions at a private tertiary care hospital in south India. J Res Med Sci. 2011; 16:16-25. PMid:21448378 PMCid:PMC3063432 
5. Ramesh M, Pandit J, Parthasarathi G. Adverse drug reactions in a south Indian hospital-their severity and cost involved. Pharmacoepidemiol Drug Saf. 2003; 12:687-92. https://doi.org/10.1002/pds.871 PMid:14762985

6. Paudel S, Chetty MS, Laudari S, Subedi ND. Adverse drug reactions of antihypertensive agents at tertiary care hospital in central Nepal. JCMS Nepal. 2017; 13(2):2849. https:// doi.org/10.3126/jcmsn.v13i2.16463
7. Eisazaei A, Vithya T, Hiremath SRR, Prasad S. Incidence and assessment of adverse drug reactions at a tertiary care hospital. WJPR. 2017; 6(12):967-8.

8. Khurshid F, Aqil M, Shamshir Alam M, Kapur P, Pillai KK. Monitoring of adverse drug reactions associated with antihypertensive medicines at a university teaching hospital in New Delhi. Daru. 2012; 20(1):34. https://doi.org/10.1186/20082231-20-34 PMid:23351598 PMCid:PMC3555729 